\title{
Analysis of the Mechanical Behaviour of an 11.5 T Nb3Sn LHC Dipole Magnet according to the Ring Collar Concept
}

A. den Ouden, H.H.J. ten Kate, D. ter Avest University of Twente, Applled Superconductivity Centre P.O. Box 217, 7500 AE Enschede, The Netherlands

Abstract-According to the CERN-LHC reference design, 10 tesla twin-aperture NbTi dipoles will be built with split collars that enclose both apertures. As part of the development program towards an experimental 11.5 tesla $\mathrm{Nb}_{3} \mathrm{Sn}$ LHC dipole magnet, the mechanical implications of an alternative collar concept have been studied with a finite element analysis. In this concept ring shaped collars are shrunk on each finished single aperture coil, thus providing the necessary room-temperature prestress. This system results in a major improvement of the stress distribution in the collars. Introduction of friction at the sliding planes can cause reopening of the gap between the yoke halves during excitation. This depends strongly on the value of the friction coefficients.

\section{INTRODUCTION}

As part of the development program towards the CERN Large Hadron Collider several $1 \mathrm{~m} 10$ tesla twin-aperture NbTI dipole model magnets have been built or are under construction. The principal mechanical and electromagnetic parameters of these magnets are referred to as the CERN-LHC reference deslgn [1]. The mechanical support structure around the coils has to ensure proper control of the Lorentz forces within the following marginal conditions :

1. the displacements of the conductor blocks are less than $20 \mathrm{um}$ to minimize fleld distortion

2. In all materials the equivalent stresses never exceed the yield stress,

3. contact between the yoke halves is maintained during excitation.

4. tangential compressive stress in the coils is preserved after excitation,

5. maximum stress in the windings is kept within the safety margins of the specific superconductor.

According to the detailed dimensional specifications of the reference design, these requirements can be met under the rather severe assumptions, that the split collar behaves like a closed, rigld structure and friction is negligible. During the design process of an 11.5 tesla $\mathrm{Nb}_{3} \mathrm{Sn}$ twin-aperture LHC dipole these assumptions have been reconsidered.

The rigidity of the split collar is at stake at the location where the two parts of the collar meet. Here, the largest stresses are expected ( $f$ 1g.1a).

Previous calculations have shown, that in this region plastic flow of the locking rods can hardly be prevented. This apparently results in a decreasing prestress in the colls. At the same time the coils are incompletely enclosed by the collars in the very same region, thus reducing the support area between coils and collars. Besides, the stacking sequence of the collar plates implies that only every second plate supports the colls properly. Another less favorable property of the

Manuscript received at 24 fune 1991. This research in the program the Foundation for Fundamental Research on Matter (FOY) has (STV)
W. van Emden, C. Daum

National Institute for Nuclear and High Energy Physics P.O.Box 41882, 1009 DB Amsterdam, The Netherlands

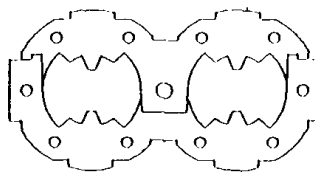

a)

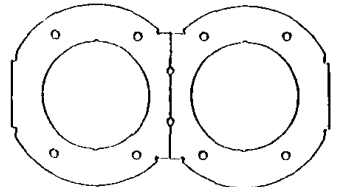

b)
F1g. 1. Proposed collar system for LHC dipoles. a) split collar according to the CERN-LHC reference design, b) ring shaped collar.

split collar system appears during the collaring process. In order to lock the collars, an external compressive force is applied, which is taken over only partially by the locking rods, or keys, due to the mechanical tolerances of the keyholes and the elasticity of the collar plates. So to obtain sufficient prestress these effects necessitates undesirable overstressing at room temperature.

On the contrary, a ring shaped collar as in fig. 1b encloses the colls completely and can be shrunk on a single impregnated coil, that is finished as a smooth cylinder. This collar concept enables a more controlled buildup of prestress and does not suffer from local peak stresses.

Though between the interfaces of yoke and outer shrinking cylinder, yoke and collar and collar and coils, sheets of copper-beryllium are inserted to reduce friction, no proper analysis of the effects of friction on the mechanical behaviour has been reported up to now. Regarding the laminated structure, the anticipated two-dimensional sliding and the relatively high contact stresses, a thorough examination of this subject is demanded.

A finite element analysis with the ANSYS code has been performed to examine the mechanical implications of both the use of a closed collar and the introduction of friction at all sliding planes.

\section{GENERAL LAYOUT OF THE 11.5 T MAGNET SYSTEM}

Regarding the current carrying capacity of ECN $\mathrm{Nb}_{3} \mathrm{Sn}$ cables, it has been decided to increase the target field for our experimental LHC dipole to 11.5 T [2]. A general reconsideration of all design parameters has resulted in two major changes in the system design. First, taking into account the specific properties of the superconductor, optimization of the cable dimensions has lead to a different conductor lay-out, as reported earlier [3]. The main electromagnet1c characteristics of the $11.5 \mathrm{I}$ dipole are summarized in table 1.

The second important deviation from the reference design concerns the support system surrounding the coils. Leaving the principle mechanical functions of the structural elements of the CERN-LHC aesign unchanged, some alterations of the mechanical layout are necessary to meet the conditions when ring shaped collars are used and the target field is increased to 11.5 tesla. The proposed structure is depicted in $f 18.2$ and will be discussed in detail, together with the modified assembly procedures.

$0018-9464 / 92 \$ 03.00$ (C) 1992 IEEE 
332

Table 1.

Main characteristics of the $11.5 \mathrm{Nb}_{3} \mathrm{Sn} \mathrm{LHC}$ dipole magnet.

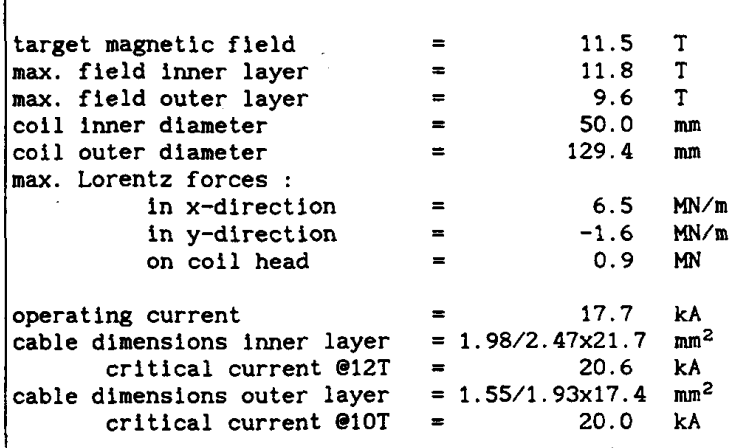

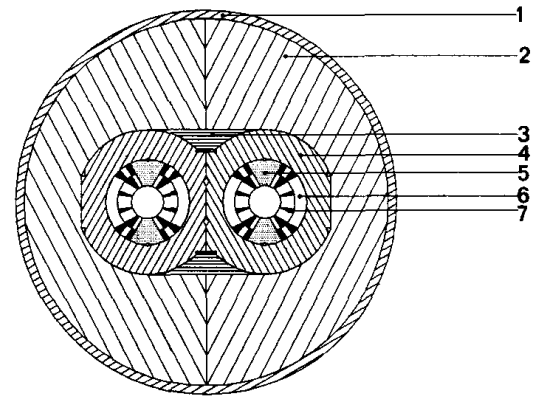

F1g. 2. Proposed structural design for the $11.5 \mathrm{~T} \mathrm{Nb}_{3} \mathrm{Sn}$ LHC dipole magnet. 1=outer cylinder, $2=y o k e$. 3=yoke insert, $4=r i n g$ collar, 5=pole insert, $6=$ conductor block, $7=$ copper wedge.

In the case of ring shaped collars, the only way to achieve prestress in the colls at room temperature is to shrink the collar plates on a single coll. Therefore the colls have to be finished as smooth cylinders, so the insert pieces that were part of the original split collars have been replaced by separate pole inserts. To enable sliding between this insert and the coll layers, that will be impregnated simultaneously, the pole face of the second layer is adjusted to the inner layer by placing another copper spacer at the top. The coll halves will be kept together by a tightly wound copper-beryllium tape of $0.4 \mathrm{~mm}$ thickness, thus providing a sliding plane between colls and collars.

The outer dlameter of the aluminum alloy collars has been increased to $110 \mathrm{~mm}$ to keep the stresses below the yield stress. The required prestress in the colls is obtained by heating the collar plates up to $225^{\circ} \mathrm{C}$ and shrink them on the coils. For an increase of the limited radial assembly space of $0.2 \mathrm{~mm}$, a higher temperature would be required. However, this might degrade the mechanlcal strength of the aluminum alloy.

After aligning the two collared single aperture coils with the central rods, the yoke assembly is straightforward. The outer dimensions of the yoke have been adapted to the increased outer dlameter of the collars in order to prevent saturation in the azimuthal plane. Contact between collar and yoke has been reserved explicitly to the vertical outer planes and the horizontal planes below the triangular yoke insert pleces. In this way the horizontal stiffness of yoke and shrinking cylinder is transferred efficiently to the collars, provided that the circumferential interfaces are given enough space to stay open after excitation.

Finally, in order to meet the outer LHC dimensions, the outer shrinking cylinder will be made of stainless steel, with a wall thickness of only $13.5 \mathrm{~mm}$.

\section{FINITE ELEMTENT ANALYSIS OF THE MECHANICAL STRUCTURE}

The mechanical analysis of the adapted structure of fig. 2 has been performed using the ANSYS code. Initially, a parameter optimization has been carried out to obtain proper performance of the structure and to find the right material for the pole inserts, ignoring friction and dimensional tolerances. Stainless steel appears to be a proper choice for the pole inserts. Its thermal contraction and its high Young's modulus match well the properties of coils and collars.

\section{A. Analysis without friction}

The result of this first computation is summarized in table $2(\mu=0)$, in which $\sigma_{\text {Eqmax }}$ represents the maximum Von Mises-stress and $\bar{\sigma}_{0}$ the average prestress on the coll at the pole face. Fig. 3 explains the directions of the forces and the positions of the polnts of interest.

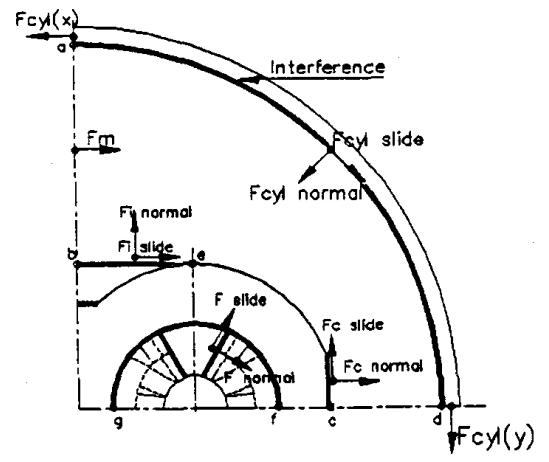

Fig. 3. Location of the forces and points of interest as used in the text. Bold lines represent the interference layers where friction is included.

As expected the highest Von Mises-stress in the colls occurs in the azimuthal plane at the inner radius. Although the quantitative $\mathrm{J}_{\mathrm{c}}$-degradation of $\mathrm{Nb}_{3} \mathrm{Sn}$ cables under transverse stress is not well defined yet, preliminary results indicate that a maximum of $150 \mathrm{~N} / \mathrm{mm}^{2}$ seems reasonable as design parameter [4].

These computed results show the rellabllity of the support structure. It should be noted however, that the outcome of the computations strongly depends on the tolerances and the exact values of the material properties. For instance, the Young's modulus is assumed homogeneous throughout the windings, whereas a radial dependence can be expected. At the same time, the Young's modulus of the impregnated conductor packages is not well known yet. First experiments on impregnated stacks of comparable rectangular ECN SULTAN cable have shown values between 16 and $21 \mathrm{kN} / \mathrm{mm}^{2}$. For the case described above a value of $20 \mathrm{kN} / \mathrm{mm}^{2}$ has been used. Fig. 4 shows the influence of the Young's modulus on the prestress at the pole face and the maximum coll stress. When the Young's modulus of the coll is less than $15 \mathrm{kN} / \mathrm{mm}^{2}$, the prestress buildup will be insufficient to guarantee contact along the entire pole plane at full excitation. This certainly is the case 


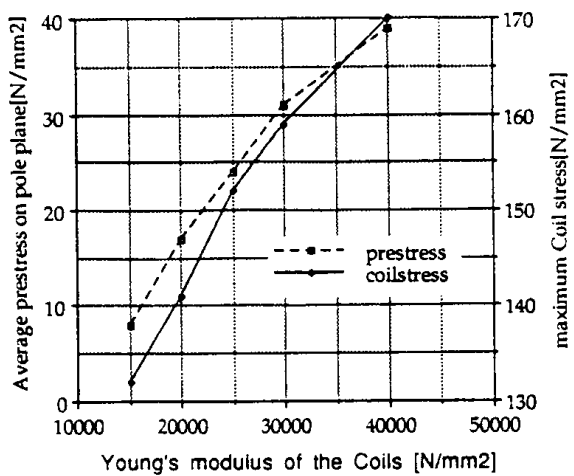

Fig. 4. Relation between the Young's modulus of the coll and the maximum coil stress and the normal stress at the pole plane at full excitation.

after the heat treatment of the coils, when they are annealed at $700{ }^{\circ} \mathrm{C}$ for several days to form the $\mathrm{Nb}_{3} \mathrm{Sn}$. The yield stress of the copper matrix is reduced to about $35 \mathrm{~N} / \mathrm{mm}^{2}$. To avoid plastic deformation and a corresponding reduction of prestress, work hardening of the impregnated coils ( by pressing them to their final dimensions) before collaring seems necessary. Though a higher average prestress naturally results in a higher maximum coil stress at the inner radius of the azimuthal plane, the latter shows a steeper dependence on the Young's modulus due to an additional contribution from the bending of the coils.

\section{B. Interface modeling including friction}

The structural analysis of the effects of friction requires to redefine the properties of the interface elements between adjacent surfaces that may slide relative to each other. These interference layers are also shown in $\mathrm{fig} .3$.

In the finite element model, sliding with friction is a conditional process of the interface elements. A proper description of friction requires a correct modeling of the normal stiffness $K_{n}$ and the tangentlal stiffness $K$, in order to avold numerical problems. An acceptable definition is given by:

$$
K_{n}=\frac{E \cdot A}{H} \text { and } K_{s}=\frac{G \cdot A}{L} \text {. }
$$

with $E=$ young's modulus, $G=s h e a r$ modulus, $A=a r e a$ around the interface element, $H=h e i g h t$ of the element and $\mathrm{L}=$ tangential length of the element.

In the definition a friction coefficient $\mu$, no sliding occurs if the tangential force $F_{s}<\mu . F_{n}$, where $F_{n}$ is the normal force at the element. However, if $F_{s} \geq \mu . F_{n}$ sliding occurs. This process is non-conservative in the sense, that the outcome is load history dependent. Convergence problems, expressed as oscillations of the element status, can arise when $\mu$ is small. This can be avolded by a subdivision in load steps. In our case this means, that the load step scheme has to be subdivided into 13 steps, 9 for the excitation and the resulting 4 for collaring, clamping, outer cylinder and cool-down. For $\mu=0.2$ the excitation step has to be subdivided in 16 load steps to reach convergence.

\section{Coefficient of static friction}

Though sheets of copper-beryllium are inserted as interface layer between all the sliding planes no exact knowledge about the friction coefflcient for the metal pairs considered is available. Regarding the, in most cases contradictory, reported values for comparable metal pairs, an estimation of the static friction coefficient between 0.3 and 0.6 is realistic $[5],[6]$. This uncertainty in the friction coefficient is determined by rather qualitative parameters as surface roughness, lubrication, atmosphere, temperature and normal stress. In order to examine its influence on the dynamics of the magnet system, computations with $\mu=0.3$, 0.4 and 0.6 have been carried out and the results are compared to the case $\mu=0$.

\section{Results including friction}

Though for all values of the friction coefficient full computations with respect to displacements and stresses have been performed, for clarity only for an intermediate value of $\mu=0.4$ the results after each load step are presented in table 2 , together with the resulting forces and stresses after excitation for all values of $\mu$. For these calculations the dimensional parameters are the same as in the optimized case for $\mu=0$.

Table 2.

Resulting fores and stresses in the structural elements after various load cases and with different friction coefficients $\mu$. (col=collaring, cla=clamping, ocyl=outer cylinder, codo=cool down, excl=excitation)

\begin{tabular}{|c|c|c|c|c|c|c|c|c|c|c|c|c|c|c|c|c|}
\hline \multirow[b]{2}{*}{$\begin{array}{l}\text { Load } \\
\text { case }\end{array}$} & \multirow[b]{2}{*}{$\mu$} & \multirow{2}{*}{\begin{tabular}{|c|} 
inser $t$ \\
$\sigma_{\text {Eqmax }}$ \\
$\mathrm{N} / \mathrm{mm}^{2}$ \\
\end{tabular}} & \multicolumn{4}{|c|}{ coils } & \multicolumn{3}{|c|}{ collars } & \multicolumn{4}{|c|}{ yoke } & \multicolumn{3}{|c|}{ outer cylinder } \\
\hline & & & $\begin{array}{c}\sum F_{n o r} \\
N\end{array}$ & $\begin{array}{c}\Sigma F_{S 11} \\
N\end{array}$ & $\begin{array}{r}\sigma_{\text {Eqmax }} \\
\mathrm{N} / \mathrm{mm}^{2}\end{array}$ & $\begin{array}{c}\bar{\sigma}_{0} \\
\mathrm{~N} / \mathrm{mm}^{2}\end{array}$ & $\begin{array}{c}\Sigma F_{\text {Cnor }} \\
\mathrm{N}\end{array}$ & $\begin{array}{c}\Sigma F_{\text {CSII }} \\
N\end{array}$ & $\left\{\begin{array}{c}\sigma_{\text {Eqmax }} \\
\mathrm{N} / \mathrm{mm}^{2}\end{array}\right.$ & $\begin{array}{c}\Sigma F_{m} \\
N\end{array}$ & $\begin{array}{c}\Sigma F_{\text {inor }} \\
N\end{array}$ & $\begin{array}{c}\Sigma F_{1 \mathrm{~s} 11} \\
\mathrm{~N}\end{array}$ & $\begin{array}{r}\sigma_{E q \max } \\
\mathrm{N} / \mathrm{mm}^{2}\end{array}$ & $\begin{array}{c}F_{c y \mid x} \\
N\end{array}$ & $\begin{array}{c}F_{\text {cyl }} . \\
N\end{array}$ & $\begin{array}{l}\sigma_{\text {Eqmax }} \\
\mathrm{N} / \mathrm{mm}^{2}\end{array}$ \\
\hline $\operatorname{col}$ & 0 & 92 & 1393 & - & 63 & 35 & & - & 103 & - & - & - & - & - & - & - \\
\hline cla & 0 & 121 & 1438 & - & 61 & 36 & 100 & - & 92 & - & - & - & 20 & - & - & - \\
\hline ocyl & 0 & 448 & 1941 & - & 108 & 49 & 1292 & - & 124 & - & 892 & - & 192 & 1291 & 1296 & 130 \\
\hline codo & 0 & 285 & 2724 & - & 116 & 68 & 283 & - & 161 & 1749 & 503 & - & 121 & 2033 & 2043 & 217 \\
\hline exci & 0 & 70 & 822 & - & 138 & 21 & 1803 & - & 223 & 268 & 1109 & - & 180 & 2073 & 2082 & 219 \\
\hline $\mathrm{col}$ & 0.4 & 113 & 1490 & 142 & 67 & 37 & - & - & 111 & - & - & - & - & - & - & - \\
\hline $\mathrm{cla}$ & 0.4 & 116 & 1562 & 120 & 64 & 39 & 100 & 8 & 110 & - & - & - & 20 & - & - & - \\
\hline ocyl & 0.4 & 329 & 2119 & 177 & 79 & 53 & 1068 & 78 & 123 & - & 671 & 244 & 114 & 1312 & 2370 & 183 \\
\hline codo & 0.4 & 386 & 2901 & 459 & 142 & 73 & 331 & 25 & 202 & 1146 & 282 & 36 & 121 & 1514 & 2578 & 207 \\
\hline exci & 0.4 & 97 & 1068 & 407 & 153 & 27 & 1772 & 292 & 176 & 0 & 762 & 90 & 166 & 1682 & 2810 & 215 \\
\hline exci & 0 & 70 & 822 & - & 138 & 21 & 1803 & - & 223 & 268 & 1109 & - & 180 & 2073 & 2082 & 219 \\
\hline exci & 0.3 & 78 & 1031 & 281 & 150 & 26 & 1825 & 285 & 203 & 0 & 850 & 69 & 175 & 1752 & 2607 & 212 \\
\hline exci & 0.4 & 97 & 1067 & 407 & 153 & 27 & 1722 & 292 & 176 & 0 & 762 & 90 & 166 & 1682 & 2810 & 215 \\
\hline exci & 0.6 & 178 & 995 & 539 & 166 & 25 & 1720 & 295 & 168 & 0 & 606 & 167 & 166 & 1554 & 3255 & 254 \\
\hline
\end{tabular}


334

Friction manifests itself already during the collaring process. Because the insert does not slide along the pole plane and is stuck to the coll face, $\sigma_{0}$ increases with $7 \%$.

After clamping the yoke halves, the different parts do not slide and friction has hardly any effect on this load case.

While shrinking the outer cylinder on the yoke, these parts will slide relative to each other because of the open yoke gap. Beside the rather high normal forces between them, a relatively high sliding force develops. which is equal to $F_{\text {cylx }}-F_{\text {cyly }}$. Due to this large friction force and the sliding force in the plane $B E$ as well, the yoke moves less Inward and bends less, which results in a reduced normal force $F_{1 \text { nor }}$ on the $B E$ plane.

During cooling the whole structure down to $4.2 \mathrm{~K}$ the gap between the yoke parts indeed closes, but the mating force $\Sigma F$ is $34 \%$ smaller than in the case $\mu=0$. This already indicates clearly the unfavorable effect of friction on the relative motion of the parts.

After excitation, the reduced mating force $\Sigma F_{\text {m }}$ appears to be insufficient to keep the gap closed. As can be seen in table 2, $\Sigma F$ reduces to zero at excitation and between the yoke halves a gap of $34 \mu \mathrm{m}$ appears.

Table 2 also shows an increase of the average contact stress $\bar{\sigma}_{0}$ when friction is taken into account. However, regarding the winding layers separately, the average contact stress at the outer layer is $47.5 \mathrm{~N} / \mathrm{mm}^{2}$, whereas that at the inner layer amounts only $10.4 \mathrm{~N} / \mathrm{mm}^{2}$. Without friction these values are 22.7 and $19.2 \mathrm{~N} / \mathrm{mm}^{2}$ respectively. The friction forces along the pole plane clearly result in an inward bending of the colls, causing contact loss between the inner layer and insert, which is a highly undesirable situation. This means, that desplte the acceptable value of the mean pole stress $\bar{\sigma}_{0}$, parts of the layers show a too large displacement, especially in the very region in the inner layer where the highest magnetic field is expected and the safety margin for the $\mathrm{Nb}_{3} \mathrm{Sn}$ conductors is small.

This same inhomogeneous stress distribution along the pole face, and in the coll package as well, introduces shear stresses between the winding layers of about 28 $\mathrm{N} / \mathrm{mm}^{2}$. Though the whole coil package is impregnated, the shear stresses probably exceed the shear strength of the insulation layer between the winding layers, which might cause cracks, or worse, the winding layers will be torn apart. In the frictionless case this phenomenon is less serious. It is interesting, that by replacing the copper wedges in the windings by wedges, that have exactly the same material properties as the winding layers, the stress distribution is significantly homogenized, thus reducing the shear stresses. At the same time the maximum coll stress decreases with about $30 \%$

The last four rows of table 2 display the resulting forces and stresses after excitation for $\mu=0,0.3,0.4$ and 0.6 respectively. As expected, the maximum stress in the colls in the azimuthal plane at the inner layer increases with increasing $\mu$, to rather large values of 153 and $166 \mathrm{~N} / \mathrm{mm}^{2}$ for $\mu=0.4$ and 0.6 respectively, which exceed the tolerable maximum of $150 \mathrm{~N} / \mathrm{mm}^{2}$.

Finally, an increase of $\mu$ reduces $F_{\text {cylx }}$ due to the development of the sliding force between yoke and outer cylinder, whlch results in opening of the yoke gap at full excitation.

\section{OPTIMIZATION OF THE STRUCTURE}

Including friction in the mechanical dymamics shows the tendency towards a reduced mating force of the yoke halves and contact loss between the inner winding layer and the insert. For $\mu=0.4$ a theoretical adaptation of the gap parameters of the yoke can be deduced. Whereas at room temperature the gap amounts 0.456 and $0.337 \mathrm{mn}$ at point $A$ and $B$ in fig. 3 respectively for optimal performance when $\mu=0$, these values have to be adapted to
0.407 and $0.290 \mathrm{~mm}$ to ensure a closed gap at excitation in the case $\mu=0.4$. At the same time contact loss at the pole plane can be prevented if the pole angle of the stainless steel insert is tilted with 0.04 degrees, corresponding to a beveling of that face with $0.05 \mathrm{~mm}$. These values indicate the sensitivity of the structure to dimensional tolerances. However, from this point of view it seems possible during the assembly of the system to use well-deflned shims at both surfaces if measurements of the dimensions ( after impregnation and collaring) necessitates these adaptations.

It has been shown that coverage of sliding surfaces with teflon sheet or MoS powder c.q. grease, even under cryogenic conditions and high contact stresses, can reduce the friction coefficient to about 0.1 [7], [8]. A low friction coefficient can prevent slip-stick and reduces the uncertainty margins of this parameter.

\section{CONCLUSIONS}

A finite element mechanical analysis of the support structure of a fully impregnated $11.5 \mathrm{~T}$ twin-aperture $\mathrm{Nb}_{3} \mathrm{Sn}$ LHC dipole confirms the feasibility of $\mathrm{ring}$ shaped collars around each coil and proves their favorable properties in comparison with the collar system proposed for .LHC dipole magnets. This collar system will be incorporated in this magnet system. Computations including friction at all the sliding surfaces show that two major problems arise in the mechanical dynamics. Assuming a reasonable value for the friction coefficient between 0.3 and 0.6 , the yoke halves loose contact during excitation and the inner layer loses contact with the pole insert. At the same time the shear stresses between the winding layers increase to unacceptably high values above the shear strength of the insulation layer.

\section{REFERENCES}

[1] R. Perin. The CERN LHC magnet team. "Flrst results of the high fleld magnet development for the Large Hadron Collider". Proc. MT-11 Tsukuba Japan 1989, Ed. T. Seklgush1, S. Shimamoto, Elsevier Appl. Science, London, (1990), p. 42-47

[2] H.H.J. ten Kate, A. den Ouden, D. ter Avest, S. Wessel, R. Dubbeldam, W. van Enden, C. Daum, M. Bona, R. Perin, "Development of an experimental $10 \mathrm{~T} \mathrm{Nb} \mathrm{Sn}$ dipole magnet for the CER LHC", Proc. ASC90 Snowmass, (1990)

[3] D. ter Avest, H.H.J. ten Kate, L.J.M. van de Kiundert, "Optiaizing the conductor dimensions for a $10-13$ I superconducting dipole magnet", ProC. ASC90, Snownass, paper LCP31, (1990)

[4] H.H.J. ten Kate, H. Boschman, H. Wel Jers, S. Wessel, L.J.M. van de Xlundert, "The reduction of the critical current in $\mathrm{Nb}_{3} \mathrm{Sn}$ cables under transverse forces", unpublished, presented paper DB-4 at MT12, Leningrad, (1991)

(5) E. Rabinowlcz, "The determination of compatiblilty of metals through static friction tests". Proc. of the ASLY/ASLE lubrication Conference. CIncinatt1, Oh10. (1970)

[6] F.D. Fuller. "Frletion" in Marks' Handbook for Mechanical Engineers, 8th ed.. T. Baume1ster, Ed., MeGraw-H111, New York, (1978)

(7) R.L. Tobler, "A Review of antifriction materials and design for cryogenic environments". Advances in Cryogenic Englneering (materials), vol. 26, pp. 66. (1979) in Cryogenle

[8] J.L Lizon, "Comparison between various lubricants at cryogenic temperatures in a vacuum". Advances in Gryogenic Englneering (materials), vol. $36,(1990)$ 